\title{
Jakuta Alikavazovic, La blonde et le bunker
}

\section{Derrière le miroir...}

Marjorie BROUSSIN, Université Lumière Lyon 2

Chloé RICHER, École Normale Supérieure de Lyon

Tatiana TuMANOvA, Université Lumière Lyon 2

«Que mon talon l'écrase...

Et brise le miroir où tu t'étais mirée $[\ldots] »$

Henri de Régnier, Les médailles d'argile, «L'infidèle »

\section{La blonde et le bunker, sur les traces d'un roman}

«Le roman peut se lire comme une variation, façon roman noir, sur le mythe d'Eurydice aux Enfers. À la fois en raison du désir de conservation (le refus de la mort) et de la perte provoquée par le fait de voir, de se retourner. » Lorsqu'elle évoque le mythe d'Eurydice aux Enfers, qui constitue le sous-bassement de l'intégralité de son dernier roman, La blonde et le bunker, l'écrivaine Jakuta Alikavazovic se réfère au tableau de Jean-Baptiste Camille Corot intitulé Orphée ramenant Eurydice des Enfers ${ }^{1}$. Sa formation d'historienne de l'art nourrit une écriture dynamique et ludique, qui constitue une chambre de la mémoire autant qu'un jeu avec les codes de notre époque. Ainsi, dans La blonde et le bunker, paru en 2012 aux éditions de l'Olivier, c'est au sein d'un trio amoureux (Anna la femme fatale, John l'époux et Gray l'amant) qu'elle remet en scène John Volstead, l'auteur des Narcissiques anonymes (découvert dans les Corps volatils en 2007) dans une narration aux allures d'enquête policière. C'est le nouvel arrivant, l'amant, qui se fait le relais de l'Orphée de Corot et prend le lecteur par la main pour tenter de comprendre l'histoire d'une mystérieuse photographie qu'Anna n'a de cesse de détruire, et qui trône dans leur maison aux allures de bunker. Mais le limier doit bientôt se lancer dans une autre quête, celle que lui laisse John à sa mort: découvrir la nature et la composition d'une collection d'art mythique, dont l'existence même reste à prouver.

Le roman que nous propose cette fois la romancière française, née en 1979, après le prix Goncourt du premier roman avec Les corps Volatils, a remporté la mention spéciale du jury Wepler. Et l'œuvre perturbe en effet tous nos repères, réalisant les aspirations de son auteure: «L'originalité dans la répétition. Le déjà-vu comme condition du sens, et paradoxalement comme irruption de la nouveauté. » Impossible de se raccrocher longtemps au désir d'élucidation, moteur du roman noir, tant la narration joue du speculum, à la fois jeux de regards et de miroirs, et spéculations de toutes sortes. Et pourtant, Jakuta Alikavazovic a 
éveillé notre curiosité et notre instinct de détective. C'est pourquoi nous vous proposons de nous suivre dans cette enquête, sur les traces du récit, avec pour seul indice celui qu'a laissé l'auteure derrière elle: «L'un des thèmes de La blonde et le bunker (comme de tous mes livres) est la disparition $»^{2}$.

\section{Image(s), reflet(s) et représentation(s) : variations sur un mythe}

\section{Spéculation et spécularité : une enquête réversible}

«Il ne s'attend pas à trouver la fin du monde, ici, mais il ne s'attend pas non plus à ne pas la trouver. » (Robert Coover)

Empruntée à l'auteur américain Robert Coover, l'épigraphe de La blonde et le bunker de Jakuta Alikavazovic place dès les premières pages le roman sous le signe de la spécularité. Cette citation, qui pose un possible et son inverse, fonctionne selon le principe de la réversibilité et marque l'impossibilité de poser un sens stable, une réponse établie : tout, ou rien, est possible. Reflétée en elle-même par effet de parallélisme («il ne s'attend pas à trouver la fin du monde ici, mais il ne s'attend pas non plus à ne pas le trouver. »), la phrase est construite selon une structure spéculative, aux deux sens du terme, à la fois miroir et réflexion sur une recherche possible. La matrice même du roman - fonctionnant sur la réversibilité - est ainsi posée dès l'incipit, et engendre une thématique qui sera reprise et développée tout au long du récit. La dimension spéculaire de l'œuvre est particulièrement mise en lumière par la reprise de l'épigraphe, modifiée et insérée dans le corps du roman sous la forme d'un commentaire extrait d'un « Manuel de survie nucléaire » :

Selon l'auteur, deux prémisses de raisonnement sont faux, fort répandus, et particulièrement dangereux. La première erreur consiste à penser qu'une catastrophe d'une telle ampleur serait si désastreuse qu'elle en devient impensable («la fin du monde n'aura pas lieu»). La seconde erreur est de croire qu'une guerre nucléaire sonnerait, de façon inévitable, le glas de l'espèce humaine (« la fin du monde aura lieu »). (135)

Cette analyse entraîne un commentaire de la part de l'auteur des Narcissiques anonymes qui éclaire le fonctionnement de l'enquête placée au centre du roman : « [...] (mais je me demande moi, ce que c'est que cette fin du monde qui aura, et n'aura pas, lieu)». Dans cette parenthèse, se fait entendre la voix d'un personnage-écrivain commentant un ouvrage a priori scientifique et réécrivant en réalité l'épigraphe qui ouvre le roman que le lecteur tient dans les mains : double structure de miroirs, où le personnage-écrivain accomplit le même geste que l'auteur du roman en venant insérer dans son texte (son roman à venir) une réflexion qui renvoie à celle qui ouvre le récit cadre. Fonctionnement spéculaire, donc, mais également 
indice sur le degré d'existence et de véracité à accorder aux événements présentés dans le roman. La question que se pose John concernant la plausibilité d'un événement qui peut ou peut ne pas arriver, redouble, semble-t-il, le questionnement du lecteur quant à l'existence de la collection Castiglioni, dont le degré d'existence et de véracité ne cesse d'être mis en doute tout au long du récit, et dont on apprendra finalement qu'elle n'existe pas, et qu'elle n'est qu'un leurre soigneusement mis en place par Vivian ${ }^{3}$.

Le fonctionnement spéculaire du roman est également mis en lumière par la manière dont se déroule l'enquête menée par Gray. Les premières pages du roman laissent attendre un déroulement selon le modèle codé et connu du roman d'enquête, modèle enclenché par la mention de la «ligne» dont hérite Gray: «Gray héritait d'une ligne. [...] Gray: elle prétendra avoir tout détruit. Elle mentira. Je ne suis pas sûr d'en savoir plus (Collezione Castiglioni ?). Gray se sentit interpellé. Il sut immédiatement de quoi le mort l'entretenait » (Alikavazovic 12). Le testament met ainsi l'intrigue en marche en jouant sur les effets de mystère. Ce «mort » que Gray semble bien connaître, est encore un inconnu pour le lecteur, et son legs prend la forme d'une énigme, énigme rendue explicite par son insertion sous forme de parenthèse interrogative au sein de la fameuse ligne d'héritage. Un mystère entoure également l'identité du personnage féminin uniquement désignée par le pronom personnel, ce qui suppose qu'elle est une connaissance commune des deux personnages masculins. Qui est ce « elle»? Qui est le mort? Qu'est-ce que cette «Collezione Castiglioni » dont l'existence est, dès sa première mention, mise en doute par la forme interrogative ? Autant de questions qui constituent les prémisses d'un roman d'enquête et créent le mystère servant à construire l'intrigue.

\section{Miroir, mon beau miroir ...}

Pourtant, de roman d'enquête il n'est pas question avant la deuxième partie du roman où se déroulent enfin sous nos yeux les différentes étapes de la recherche menée par Gray. Le départ pour Venise, annoncé dès la page 12 («Gray prit sa ligne d'héritage, ou son héritage d'une ligne - ce qui revient au même - et quitta la ville »), n'est effectif dans la diégèse qu'à partir de la moitié du roman ${ }^{4}$. Cet effet de retardement est imposé par la structure du récit, la première partie constituant une analepse centrée sur les différentes étapes de la relation entre Gray et Anna, puis sur les rencontres successives entre Gray et John. L'histoire du trio amoureux se substitue ainsi à l'enquête sur la collection à laquelle le lecteur s'attend, après la mise en haleine des premières pages. Pour autant, la dimension inquisitoriale n'est pas occultée dans cette première partie puisque le récit progresse par alternance de chapitres narrant les relations entre les trois personnages et de chapitres constitués de réflexions sur la 
conservation des œuvres d'art. Des indices de l'existence de la Collection, sous forme de courts extraits d'ouvrages scientifiques attribués au professeur Jasper Warski ${ }^{5}$, sont ainsi distillés tout au long de la première partie du roman et maintiennent éveillée la curiosité du lecteur, alors même que l'enquête à proprement parler n'a pas encore pris forme. La structure du roman pourrait dès lors apparaître comme une volonté de retarder le moment où Gray part à la recherche de la collection, afin de créer un effet d'attente. Mais, plus encore, il semble que le lien entre les deux premières parties se fasse selon une logique qui a beaucoup à voir avec la thématique du miroir mise en place dès le début du récit. En effet, si l'enquête se déroule tout au long des deuxième et troisième parties du roman, on remarque que la structure de la deuxième partie fonctionne comme un écho à la première partie. Là où alternaient chapitres narratifs centrés sur le trio amoureux et chapitres théoriques présentant des réflexions sur l'art et sa conservation, se succèdent désormais des chapitres plongeant le lecteur au cœur de l'enquête, au plus près des manifestations et des disparitions de la Collection, et des extraits du roman inachevé de John revenant sur son histoire d'amour avec Anna. La voix du romancier mort, disparu de facto de l'intrigue, se substitue aux réflexions $\mathrm{du}$ professeur devenu quant à lui un personnage central - vivant - , selon une logique symétrique qui permet de superposer les deux parties pour faire apparaître toute une série de reprises, de d'échos et de doubles.

\section{La puissance souterraine du mythe}

$\mathrm{Si}$, comme l'indique Jakuta Alikavazovic, le roman peut se lire comme une «variation, façon roman noir, sur le mythe d'Eurydice aux Enfers », la nature de cette variation reste à identifier pour le lecteur en quête de sens. Présentés explicitement pour la première fois dans un chapitre consacré à la collection ${ }^{6}$, la figure d'Eurydice, ainsi que le geste d'Orphée se retournant et perdant à jamais l'objet de son amour, semblent rayonner à travers l'œuvre et essaimer toute une série de re-présentations. La variation à laquelle l'auteur se livre inscrit alors le mythe comme une image fondatrice - on aurait envie de dire souterraine - au miroir de laquelle viennent se refléter un à un les personnages, les événements et les lieux.

De même que le roman est construit selon un système d'échos entre la première et la deuxième partie (la troisième pouvant, de fait, être considérée comme un épilogue), la structuration de l'espace reproduit cette partition symétrique et figure une reprise moderne du mythe orphique. La maison d'Anna et John, présentée comme un «bunker chaulé à la hâte » (Alikavazovic, 18), et construite sur « deux niveaux, rez-de-chaussée et rez-de-jardin » (18), apparaît comme une représentation de l'univers mythologique où s'opposent monde des 
vivants et monde de morts, terre et Enfers. La partie souterraine de la maison est le domaine de John, sorte de créature inconnue et inquiétante que Gray entend marcher la nuit, sans jamais l'avoir rencontré ${ }^{7}$; partie souterraine qui figure un Enfer moderne, dans lequel vit reclus un écrivain mal-aimé qui n'écrit plus rien, sorte de mort qui hante le sous-sol du monde des vivants. Mû par la curiosité et le désir d'en découvrir plus sur sa mystérieuse maîtresse, Gray finit par s'aventurer dans ce monde inférieur, effectuant tel Orphée une descente aux Enfers : «Gray, désœuvré, errait dans la maison. Il descendit trois marches qui menaient au sous-sol et aperçut la grande photographie, ou du moins son tiers inférieur [...]» (34).

Mais, à l'inverse d'Orphée, Gray n'accomplit qu'une catabase incomplète. La photographie de l'autographe frontal, placée comme un seuil entre les deux espaces, lui fait rebrousser chemin (34) et c'est à l'étage qu'il découvrira John, affairé à récupérer des livres dans la chambre qu'il occupe ${ }^{8}$. C'est que, dans cette reprise moderne du mythe, Gray n'est pas une figure orphique, mais bien plutôt son miroir inversé, son négatif - au sens photographique.

\section{Quand Orphée se fait femme ...}

Orchestrant une variation sur le mythe, Jakuta Alizavazovic s'amuse à reprendre et inverser ses éléments. Ce n'est plus un poète désespéré voulant retrouver sa bien-aimée qui descend aux Enfers, mais un amant ennuyé qui recherche la compagnie d'un ex-mari écrivain bibliomaniaque ; ce n'est plus le héros qui ramène son épouse vers le monde des vivants, mais un enquêteur malhabile entraîné dans le sillage de femmes dites «fatales » qui le conduisent dans un monde où règnent le mystère et la disparition. D'abord Anna qui, après leur première rencontre au Centre Pompidou, « ramèn[e] [Gray] chez elle » (17), jouant ainsi le rôle d'Orphée ; puis Vivian, que Gray devenu filateur suit dans les rues de Venise. Dans le dédale de la ville italienne, le jeune homme a conscience de reproduire un scénario de film noir, une «filature à l'ancienne» (181). Mais, plus encore que celui de l'enquêteur, c'est le rôle d'Eurydice que Gray endosse symboliquement en accordant ses pas à ceux de Vivian, qui le mènera - espère-t-il - vers l'horizon tant désiré de la Collection. Mythe réécrit en négatif, donc, mais également effet de miroir intra-textuel : la scène de filature renvoie comme un écho à la description de la vidéo du Corridor que le professeur cherche désespérément à retrouver'. Un enchaînement d'effets spéculaire se déploie ici puisque si Gray suivant Vivian figure Eurydice, il reproduit également en l'inversant le schéma de la vidéo, laquelle est ellemême une reprise du mythe orphique ${ }^{10}$.

Au sein de ce régime généralisé de la reprise et de la spécularité, le roman progresse 
par répétitions et inversion de scènes déjà-vues, par duplication de moments et de personnages. Si, dans la réécriture du mythe, Anna et Vivian apparaissent toutes deux comme des figurations possibles d'Orphée face à un Gray-Eurydice, elles fonctionnent également comme les deux faces d'une même figure. Anna la blonde, froide et vaporeuse, forme le pendant de Vivian la brune ; là où l'une constitue l'élément déclencheur de l'enquête (« elle prétendra avoir tout détruit »), l'autre est celle par qui la résolution - ou plutôt son absence arrive (194). Placées chacune à un pôle de l'histoire, le caractère spéculaire de leur identité est marqué par la trace qui les relie : à l'autographe frontal qui signe le lien entre Anna et John répond la trace de cambouis dont Vivian se macule le front.

$R e$-présentant des figures du mythe orphique, le roman est ainsi construit selon une logique de répétition et d'inversion, tant au point de la structure générale du récit que des déploiements de l'intrigue. Moins qu'une réécriture du mythe, c'est un écho que nous propose Jakuta Alikavazovic, un reflet au miroir duquel il convient de lire le texte, en prenant le risque de s'y perdre... comme Narcisse, autre mythe convoqué de manière ludique par l'auteur qui n'a de cesse d'interroger la question « de la répétition, des codes, et bien sûr de leur perturbation » et du «déjà-vu comme condition du sens » ${ }^{11}$. Narcisse et Orphée, deux figurations d'un regard qui se perd lui-même, ou perd l'autre; deux images des risques encourus à vouloir «trop voir $»^{12}$.

\section{Du cliché à l'anéantissement, enquêtes sur le pouvoir du regard}

\section{Que voir ? Comment voir?}

La spécularité et l'effet du miroir qui marquent le roman, amènent à envisager le rôle du regard dans la narration. À la lecture de l'épigraphe de la première partie, citation empruntée à Sophocle, on comprend que le regard jouera un rôle central dans le roman $L a$ blonde et le bunker dès qu'on ouvre le livre : «Pourquoi donc avez-vous peur, comme des enfants, avant de ne rien voir ?» (11). Il s'agit d'un regard particulier, qui est important mais qui constate plutôt une absence qu'une présence ; il cache au lieu de donner un sens à la réalité observée, il trouble et fait peur. Une menace plane, qui reste invisible mais néanmoins presque palpable.

Pour illustrer cela, il convient de s'intéresser deux personnages principaux - Anna et Gray. Anna est la femme fatale du roman ; en outre, elle est photographe et donc sa perception de la réalité est insolite et très intéressante. Quant à Gray, il est d'abord un observateur : en arrivant chez Anna et son ex-mari, il reste dans une chambre d'amis - par conséquent, le héros devient un hôte, un invité qui demeure en extérieur par rapport aux deux 
personnages mentionnés plus haut. Par la suite, Gray se transforme en enquêteur, rôle qui implique l'importance de l'observation, et par conséquent du regard.

La présentation des personnages commence par Gray, un Américain installé en France. Son histoire reste mystérieuse, rien ou presque n'est dit de son passé : on sait juste que Gray a eu un grave accident de voiture et qu'il fréquente le Centre Pompidou, lieu où il rencontre Anna. Gray la voit plusieurs fois et ensuite il la suit pour faire l'amour dans les toilettes, moment où le regard devient essentiel puisque tout se passe devant un miroir : «Elle, qui tournait le dos au miroir (hasard, prudence ou préméditation) [...] lui en revanche ne pouvait se séparer de lui-même qui se regardait faire. Il ne se reconnaissait pas, il lui semblait qu'ils étaient trois... » (16).

Dès le début Gray est un observateur : le jeune homme est face à lui-même, il est mis dans la position où il est obligé de se regarder et de se voir comme un étranger. On peut dire qu'Anna rend le personnage méconnaissable à lui-même. Ainsi, lorsque la femme décrit le visage de Gray ou le prend en photo en utilisant des lampes au sodium et des tubes fluorescents, il ne se reconnaît plus : «C'est en réalité une question de lumière [...]. D’où l'impression d'étrangeté, de perte de repères éprouvée à découvrir sous en éclairage inhabituel des lieux pourtant communs, aussi commun que son propre visage. [...] Voilà sur quoi travaillait Anna - sur les faiblesses de l'œil humain » (27).

Ainsi, le regard de cette femme s'avère très particulier: au lieu de découvrir le monde, de le montrer à travers ses photographies, elle préfère dérouter les spectateurs, les tromper. Anna choisit de prendre en photo des lieux ou des individus sous une lumière différente de l'habituelle. Il s'agit d'une lumière complètement artificielle et inquiétante, sous l'influence de laquelle Gray a l'impression que sa chambre se métamorphose en « scène de crime » (27). Le regard d'Anna transforme la réalité et la défigure.

On peut dire que cette femme cache le monde en jouant avec la lumière, elle remplace l'intérieur par l'extérieur. La lumière avec laquelle elle joue pour construire ses images photographiques appartient en propre à l'espace public, et elle la déplace pour capturer des scènes domestiques (dont des clichés de son mari « au bras cassé ») : «les lampes au sodium [...] furent utilisées dans l'éclairage urbain, principalement dans les années 1970 ; principalement en extérieur (parkings; périphériques). Les tubes fluorescents sont eux communs aux espaces publics et utilitaires » (25).

Autrement dit, le regard d'Anna rend le monde étranger par la destruction de l'espace intime, tout devient extérieur et froid, à l'image de cette femme elle-même. Selon la description psychologique qu'en fait Gray, elle aurait « la passion de l'indifférence, tout lui 
[est] égal » (79). Anna ne semble pas éprouver des émotions ou s'attacher à quoi que ce soit ; les hommes tombent amoureux d'elle mais elle demeure froide. Son ex-mari John dit à Gray qu'elle «laisse des morts derrière ». Ce détail, qui rejoint l'indifférence d'Anna et son tempérament, rapproche le personnage de la figure mythique de la Gorgone.

\section{Elle a le regard qui tue...}

D’après Max Milner, pour se représenter cette créature, il faut «pouvoir imaginer un regard neutre, sans intentionnalité [...] et qui pourtant me fixe, me dépouille de toute possibilité de réponse, me réduit à l'état de chose »(21). N'est-ce pas exactement ce qui arrive à Gray et John ? Le premier personnage n'arrive pas à se détacher d'Anna malgré la froideur dont elle fait preuve ; il reste dans sa maison sans avoir le statut de membre de famille. Il demeure dans la chambre d'amis mais semble quasiment invisible et insignifiant, puisque souvent Anna part sans le prévenir, sans dire un mot. John, quant à lui, habite le soussol, il ne voit pas la lumière du jour. C'est un écrivain qui n'écrit plus rien, et il semble anéanti par la vie avec son ex-femme puisque la seule activité qui semble le maintenir véritablement en vie consiste à cacher les négatifs de la photo d'Anna et lui, que la photographe blonde cherche à détruire. En d'autres termes, John ne semble pas avoir une vie réelle : il existe, mais sans vivre vraiment. En cela, Anna figure une sorte de Gorgone qui tue la vie autour d'elle. Mais est-elle la seule à exercer ce pouvoir morbide ? Caractérisée par l'effet de miroir, la structure du roman redouble cette image du regard tueur, en faisant de Gray le personnage par qui la mort arrive, par le regard.

Après la mort de John et la disparition d'Anna, il cherche un endroit où dormir. Gray loue l'appartement d'un étudiant qu'il habite lorsque le locataire légitime en est absent. Pourtant, très vite, l'étudiant demande à Gray de s'en aller en expliquant que désormais il se sent mal à l'aise et dort mal, il se réveille fatigué, il a des cernes. Le personnage principal essaie de trouver un autre logement comme celui-là mais les autres étudiants refusent; finalement, quelqu'un avoue que les gens disent que Gray regarde les gens dormir et que son regard porte malheur. Au début, le personnage est confus par cette explication, mais à la fin du roman, lorsqu'il se rend compte que la collection n'existe probablement pas, le jeune homme se souvient de la rumeur en repensant à son accident de voiture, à Anna et à John : «Que disait-on de lui, déjà ? [...] Son regard portait malheur : il regardait les gens mourir » (Alikavazovi 189).

Le cercle se referme là : Anna laisse des morts derrière elle, alors que Gray regarde les gens mourir. Dans les deux cas, on retrouve le regard tueur, le regard de Gorgone. Les deux personnages semblent laisser un désert derrière eux : l'espace autour d'eux est marqué 
par la disparition - de la vie, du talent d'un écrivain, de la collection. On est condamné à chercher sans trouver, à se perdre dans les traces d'une existence qui n'est jamais là.

\section{Entre enquête et quête : les spéculations du roman}

\section{De l'indice à la trace : l'évaporation du roman noir}

« Il reste des traces du roman noir dans La blonde et le bunker », affirme Jakuta Alikavazovic dans le Making-of de D-fiction. Et d'en recenser les motifs : le anti-héros, victime d'une femme fatale, son inscription au sein d'un triptyque amoureux où la mort du mari gênant flirte avec le vaudeville. Toutefois, c'est bien dans la structure du roman, conçue comme une «manière d'enquête », que le roman noir irrigue l'écriture. La couleur est donnée dès la citation en exergue, forme d'avertissement au lecteur : l'horizon que le roman ouvre est celui d'une enquête sur fond d'Apocalypse.

\section{«Une manière d'enquête»}

Gray incarne, de manière évidente, la figure avortée de l'enquêteur du roman policier. D'abord témoin des tribulations amoureuses menant à la mort de John, il en devient ensuite l'envoyé, à la recherche d'une collection disparue, dont on ignore si elle a un jour existé. Personnage itinérant - si l'on excepte la claustration momentanée dans le bunker - il relève des indices qui déterminent son cheminement: Paris, lieu de la rencontre avec Anna, prémisses d'une histoire d'amour aux allures de filature ${ }^{13}$, puis Venise, occasion de filer une autre femme, brune cette fois, dans l'espoir qu'elle le mène au terme de son enquête, c'est-àdire à la collection Castiglioni. Gray se fait l'œil suspicieux du lecteur, son relais textuel dans le bunker et au-dehors : est-ce Anna sur la photo ? En quoi consiste véritablement la collection ? Car ne l'oublions pas, «Gray [a] hérit[é] d'une ligne » directrice. Et le lecteur avec lui. Reste à savoir où celle-ci mène.

Si l'enquête semble distribuer les espaces et opposer les lieux du crime aux espaces de filature - Venise comme ligne de fuite - La blonde et le bunker échoue néanmoins au jeu du Cluedo : il faut chercher «l'originalité dans la répétition », nous dit l'auteure. Si les rôles existent (le jeune premier, les femmes fatales, le professeur, le mort), leur distribution n'en est pas moins aléatoire et instable. A l'image d'Anna, à la fois modèle et photographe, la victime peut devenir bourreau : de la mort de John, on sait seulement qu'elle s'est produite sous (par ?) l'œil de Gray, qui finit par effrayer l'étudiant dont il mène la vie inversée sans jamais le croiser, dormant chez lui le jour, vivant la nuit. L'inquiétude est tout autant créée par les autres personnages que par l'apprenti enquêteur lui-même, qui retourne les codes de la persécution propre au roman noir, en épiant le professeur. Lui-même se trouve d'ailleurs épié 
par un « on » qui inclut la communauté du narrateur et des lecteurs : «A propos de Gray et du voyage qui devait, en dernier lieu, le lancer à la poursuite de la collection, on dispose de quelques informations », lit-on page 15 . Le speculum ne s'arrête donc pas aux frontières de la page. Et tant mieux car le meurtrier court toujours...

De la déception

Finalement, La blonde et le bunker n'est pas tant le roman d'une enquête que le roman de son échec, des tribulations d'un enquêteur douteux sur fond de mystère et de soupçon : les traces du roman noir semblent plus à chercher du côté du tableau que de l'intrigue. La peinture de la petite société du bunker ne laisse pas de place à la tendresse, et l'ouverture sur un ailleurs vénitien n'offre pas plus de complaisance. Aucune issue heureuse pour l'histoire d'amour, le monde de l'art ou la recherche universitaire. Pas de lumière dans le bunker. Du roman noir, la narration conserve l'obscurité, l'absence de couleur - la blonde est obscurcie par la brune Vivian qui lui succède, Gray lui-même n'accroche pas la lumière. Le roman fonctionne à la manière d'une série de tableaux ou de clichés, décliné sur les procédés du noir et blanc. L'obscurité naît alors de la claustration et l'opposition initiale entre le bunker, imaginaire de l'enfermement, et l'extérieur promis par Venise se résout par une nouvelle réclusion. En Italie, Gray semble trouver un bunker tout neuf chez Mitzi, à la pension Ritzi, où il passe ses journées, souvent reclus dans sa chambre.

Ainsi, l'horizon d'attente du roman d'enquête est doublement déçu : au terme de l'enquête, nulle découverte. Pas de meurtrier, aucun mobile, aucune explication à la mort de John. Pas de découverte scientifique non plus, le lecteur est aussi berné que le professeur : de la collection, pas de trace... ou plutôt si : rien que des traces.

\section{Les mensonges de la collection : variations sur Eurydice}

Dans le tableau de Corot dont son roman est la variation, Jakuta Alikavazovic perçoit une étrange tristesse chez Eurydice, réticente à quitter le royaume des Enfers, en même temps que l'instant de tension entre le désir de conservation, figuré par Orphée tenant la main de sa bien-aimée tout en brandissant sa lyre, et la proximité de la perte, entraînée par le retournement à venir. Voir, c'est tuer (on se souvient des yeux revolver de Gray). Alertée par l'imminence de la disparition, dans La blonde et le bunker, Jakuta Alikavazovic se livre à des spéculations - le speculum quitte le miroir pour se faire observation, tentative de pénétrer par l'esprit - sur l'art.

L'art, entre présence et absence : la conservation et la perte

Le temps de l'intrigue semble résider dans cet instant suspendu, qui envisage déjà la perte de ce que l'on cherchait à conserver à tout prix. Cette dialectique entre présence et 
absence permet de suturer l'histoire d'amour et la recherche de la collection Castiglioni : dans les deux cas, il s'agit de trouver les moyens de conserver l'Autre (femme ou art) par tous les procédés possibles. Après avoir épuisé le processus de duplication, grâce aux multiples dédoublements que nous avons observés, condensés dans les tirages réitérés de la photographie qui met en scène John et Anna, le roman nous fait craindre la perte d'un autre objet de convoitise : la collection. Ainsi, les figures du collectionneur, tout en saturant le texte - John et sa Bibliothèque, le professeur et Vivian, Gray et les passionnés qu'il rencontre dans sa quête, in fine le lecteur aux allures de bibliomane - échouent de manière inévitable à rassembler et conserver les œuvres. Le seul mouvement que La blonde et le bunker envisage est ce retournement d'Orphée qui transmet la mort, mouvement de retour vers le passé (on ne peut voir la collection Castiglioni, sa recherche ne saurait aboutir qu'à un historique des lieux de son passage) qui entérine la destruction.

\section{La ruine ou l'art comme dégradation}

Les chapitres sur «La collection» et les «Notes sur la conservation » qui entrecoupent l'histoire d'amour semblent enregistrer cette désagrégation de l'art. Ainsi, le chapitre d'ouverture, qui porte sur la collection, est tout entier marqué par la négation : les publications sur la Castiglioni sont « rares », et ce qu'on en dit dans un essai «non daté » et «non paginé » est faux : «En aucun cas la collection n'est temporaire. Ni fugace» (Alikavazovic, 11-13). De manière similaire, les « Notes sur la conservation (1) » (23-25) ne traitent pas de l'objet annoncé, mais au contraire des « euvres détruites et leurs traces dans la littérature ». Présentes sous la forme du polyptote, la disparition et la perte marquent le texte et son champ lexical: la sculpture n'est que «fantôme », «existence spectrale », «Glacis et pigments se désagrègent », les formats sont «obsolètes et illisibles », les couleurs « virent, se dégradent, flottent ». A travers l'observation de chaque aspect de l'œuvre, il s'agit de dire que «Le temps détruit tout, avec une inventivité et une abondance de moyens qui tiennent en respect même l'esprit le plus créatif ». La disparition contamine la narration même, qui ne peut recourir qu'aux questions rhétoriques : on ne peut plus affirmer, tout reste en suspens.

Ce mouvement de disparition, qu'enregistrent les chapitres dédiés à l'art, marque aussi l'histoire d'amour. Bien plus que de constater une disparition, il s'agit alors de détruire : aux notes sur la «conservation » répondent les lambeaux de la photographie déchirée par Anna, qui veut en retrouver les négatifs pour les supprimer. De même, John organise sa Bibliothèque des Assassins, tandis que le professeur affirme son désir de détruire les tableaux d'Eurydice. Tout semble vouloir signifier cette «crise de l'art contemporain » qu'Yves 
Michaud décrit comme une «crise de la représentation de l'art» et comme «la fin de [son] utopie ». Peut-être l'art n'est-il que latence, vide. Le personnage du professeur se fait le relais textuel de cette théorie d'une œuvre qui se dérobe, que le regard ne peut fixer : l'essence de l'art réside dans «l'invisible comme principe actif et régime d'intensité » ${ }^{14}$. Entre présence et absence, la collection ne cesse d'échapper à Gray et au lecteur, sans doute parce que la rencontre n'est pas dans le lieu et à l'instant prévus.

En effet, «le principe définitionnel de la trace ou de la ruine réside dans ce fait qu'elles sont toujours incomplètes. La trace, signe d'un événement absent qui en est la cause ; la ruine, partie d'un ensemble plus vaste et disparu et aussi signe, à son tour, de l'événement qui a provoqué cette disparition » (Montalbetti 229). Pour Gray, c'est bien de cela qu'il s'agit : chercher les traces d'une collection disparue, sur les ruines d'une histoire d'amour. L'art ne peut être retrouvé, puisqu'il ne peut être vu sans être détruit. Pourtant, les personnages persistent dans leur quête alors même qu'ils la savent vouée à l'échec : «Le temps presse - selon les prévisions du professeur, la collection Castiglioni sera à Hong Kong dans huit à dix semaines. Et je crois que cette fois sera la bonne. Ou presque » (Alikavazovi 196). Une dynamique semble donc se déployer quand même, sur les ruines d'un art et d'un amour disparu, répondant à la définition de Christine Montalbetti : «cette incomplétude crée comme une dynamique de l'appel, qui invite à la restitution par le discours de ce que la ruine ou la trace donnent à apercevoir » (229).

\section{Écrire sur des ruines : l'impossible récollection ?}

Abandonnant son histoire d'amour avec Anna, Gray quitte Paris pour l'Italie et Venise, chercher une collection disparue, dans la capitale des arts déjà presque engloutie par les eaux : «Venise hors saison était parfaite, Venise et son eau qui dévorait tout» (Alikavazovic, 97). Au terme de la seconde partie, un nouvel échec, dont l'auteure elle-même semble nous donner la clé : la collection n'était pour Gray que le MucGuffin de La Blonde, l'artifice hitchcockien qui lance la recherche du héros, un artefact, voire un subterfuge de la narration. Car si Gray ne retrouve jamais la Castiglioni, c'est une autre collection que le roman offre à la contemplation du lecteur.

\section{Roman des collections et collection du roman}

La structure du roman offre au lecteur autant d'œuvres que de chapitres. En effet, l'alternance entre l'histoire d'amour et les considérations théoriques sur l'art, appliquées de manière immédiate aux chapitres concernant «la collection» - un triptyque - permet à la blonde une distribution de l'espace-livre assez similaire à celle d'une salle d'exposition sur les murs de laquelle on peut admirer les photographies de John et Anna. En effet, si une seule 
d'entre elles est digne d'être exposée, le récit prend en charge la totalité de la collection, ce qui constitue la quasi-totalité du roman de John. Pour la romancière, nul besoin des négatifs, l'écriture suffit à la mémoire. Cette forme de la variation paraît alors dicter une écriture sérielle qui se décline à l'infini, dans la structure comme dans l'énonciation. La narration semble naître de citations toujours placées en exergue, indices d'un dialogisme omniprésent. Si l'on doute des collections dont parle le roman, on ne peut qu'admirer les divers éléments du «cabinet de curiosités » que nous propose Jakuta Alikavazovic: anecdotes savantes, digressions géographiques et historiques, réécriture des mythes, etc. L'éclatement des points de vue en permet la circulation, notamment sous la forme citationnelle. Les guillemets prélèvent un discours - le modèle - pour se l'approprier ensuite. Les italiques signalent alors au lecteur que distance est prise par rapport au modèle, que la transformation a eu lieu et qu'il s'agit là d'une œuvre, d'une citation érudite, ou encore d'un scénario possible. Bref, d'un morceau de la collection. Les syntagmes circulent d'un personnage à un autre, en manière d'autocitation (n'oublions pas que John s'est rendu célèbre par l'écriture des Narcissiques anonymes), et constituent le fil d'Ariane du musée que devient notre Bunker. Ainsi, l'expression «d'une voix neutre », désignée comme telle par la narration, est actualisée, en l'espace d'un paragraphe, par trois locuteurs différents :

Dans Les narcissiques anonymes, il [John] avait utilisé à quatre-vingtsept reprises (au moins deux personnes avaient compté, crayon en main) l'expression d'une voix neutre (soixante-dix-neuf en français, car le traducteur avait parfois opté, curieusement, pour un air détaché [...]). Il avait fait la couverture de Time Magazine, il y avait une photo de lui signant le front d'une jeune femme (à tort identifiée comme sa future épouse) et Gray, lors de ses brèves études, avait entendu un jeune présomptueux se faire tancer vertement pour avoir eu le malheur de dire d'une voix neutre dans une allocution. (33-34)

De mise en abîme en mise en abîme, vous étiez prévenu...

\section{L'xil photographique}

Il n'empêche que ce procédé dialogique - lui-même décliné par les ressources du discours rapporté - permet la prise en charge, dans la narration, des paroles et discours de l'Autre. Bien plus qu'une érudition artificielle, la récollection semble dépasser l'aporie initiale de l'art. En effet, c'est cet éclatement des points de vue qui permet une saisie plurielle de l'objet exposé, et rend à nouveau possible la collection. Si le lecteur n'est qu'un second Orphée, incapable de sauver l'œuvre/Eurydice, par son regard, la variation des points de vue, comme autant de cadres ou d'angle de vue, semble rétablir, sinon son existence, du moins celle de ses manifestations. Sur le modèle photographique, le roman offre une collection d'instantanés. A défaut de capter une essence artistique, il rend présent les manifestations de 
l'art, des clichés mis de côté jusqu'aux embryons de roman à venir (statut qu'acquiert la blonde, sujet d'un récit à la fois avorté et à venir, sur la beauté fatale). L'écriture saisit le mouvement, qui est pour l'auteure ce qui reste - la trace ou la ruine - et constitue l'art, tout comme «c'est le vide du moyeu qui fait tourner la roue $»^{15}$. Finalement, la collection qu'elle nous propose, n'est pas si éloignée de cette dynamique perpétuelle qui amenait MerleauPonty à redéfinir la peinture et l'art, non pas comme un tableau abandonné au regard du spectateur, mais comme l'esprit du créateur et du visiteur, sortant de l'œil pour aller se promener sur la toile.

\section{Conclure : petite collection portative du lecteur-bibliomane}

Alors qu'il pensait naïvement être invité à suivre les traces d'un apprenti enquêteur, le lecteur de La blonde et le bunker se trouve pris au piège d'un récit qui ne cesse de se dérober, progressant non pas linéairement de découvertes en découvertes mais de manière sinueuse, de détours en reflets, de reprises en variations. Au-delà d'un simple roman d'enquête ou d'un roman d'amour, c'est une architecture subtile et élégante que Jakuta Alikavazovic propose au regard et à la sagacité du lecteur, invité moins à chercher aux côtés de Gray les preuves de l'existence - ou non - de la fameuse Collection qu'à repérer les indices d'une forme qui joue à plaisir des potentialités de la répétition. Images dupliquées, images inversées, les personnages et le lecteur sont entraînés dans un univers où le miroir construit le sens. L'effet spéculaire irrigue en effet le texte et trace une série de parallèles, au nombre desquels se distingue celui qui se dessine entre le regard de deux personnages, Anna et Gray, et celui de Gorgone. Dans les deux cas, le regard s'avère mortel, il est lié à la destruction et à la disparition. Preuve, s'il en fallait une, que la disparition occupe le centre du récit, la progression du roman marque l'évanouissement de l'enquête pour la quête ${ }^{16}$, seule possible à mener encore sur les ruines de l'art. Le texte se propose comme une succession de clichés et de séries, qui tente de saisir le réel dans la multiplicité des regards qui se posent sur lui.

Mais si l'écriture se fait presque plastique, on ne saurait toutefois parler d'un roman photographique : le visage de Gray ne renvoie pas normalement la lumière, celle créée par Anna reste artificielle. Il n'est pas possible de conserver Eurydice, de même qu'Anna ne peut rester claustrée dans le bunker. La collection ne peut saisir l'essence d'un art qui n'est que vide : ce que livre La blonde et le bunker est une récollection plus qu'une collection. Il s'agit de rassembler, sous les yeux du lecteur, les vestiges d'un art qui ne peut plus être représentation, d'en contempler les ruines. C'est alors l'écriture qui fixe la trace : sans tenter de regarder son Eurydice en face, Jakuta Alikavazovic multiplie les points de vue, comme 
autant de focales qui offrent un angle différent du précédent. Cette stratégie du regard oblique correspond très précisément à la définition de l'ironie que Philippe Hamon développe à partir d'une photographie de Doisneau, dans laquelle la vitrine d'un antiquaire joue le rôle du miroir. Rien d'étonnant donc à ce que la seule posture possible face à l'art contemporain réside dans l'ironie et l'humour - noir -, les jeux textuels, et la réécriture ludique des mythes qui irriguaient déjà la tradition des Beaux-Arts.

\section{Bibliographie}

Alikavazovic, Jakuta. La blonde et le bunker. Paris : L'Olivier, 2012.

Michaud, Yves. La crise de l'art contemporain. Paris : PUF, coll Quadridge, 1997.

Milner, Max. On est prié de fermer les yeux. Paris : Gallimard, 1991.

Montalbetti, Christine. Le voyage, le monde et la bibliothèque. Paris : PUF, coll Écriture, 1997.

\footnotetext{
NOTES

${ }^{1}$ Tableau daté de 1861 et conservé au Museum of Fine Arts, Houston, Texas, USA

2 Jakuta Alikavazovic, extrait d'un entretien sur le blog D-fiction (http://d-fiction.fr/2012/10/la-blonde-et-lebunker-de-jakuta-alikavazovic/, consulté le 12/05/13)

« Et, à ses côtés, elle chercha assidûment une collection dont elle savait qu'elle n'était rien. » (Alikavazovic 194).

${ }^{4}$ Voir le chapitre « Venise », (Alikavazovic 95).

${ }^{5}$ Voir ainsi le chapitre « Notes sur la conservation (1) - in Jasper Warki, Voir sans voir, essai sur les ouvres détruites et leurs traces dans la littérature », (Alikavazovic 23).

${ }^{6}$ Voir chapitre « La collection » (71).

7 « [...] [Gray] l'entendait marcher. La nuit venue, surtout. Il entendait des bruits de pas, sous lui, divers itinéraires d'insomniaque, des bruits de meubles déplacés » (20).

8 «Il finit par regagner sa chambre [...]. Il poussa la porte et tomba nez à nez avec John » (34).

${ }^{9}$ Dans la scène vénitienne, il est ainsi question du « souffle » de Gray qu'il cherche à « ajuster » à celui de Vivian, souffle qui constitue l'élément principal de la description de la vidéo par le professeur et permet d'identifier un second personnage, qui tient la caméra et suit l'homme vu de dos.

${ }^{10}$ En effet, l'image de la vidéo vire au noir au moment où l'homme que quelqu'un suivait se retourne pour regarder derrière lui, tout comme Eurydice disparaît au moment où Orphée rompt sa promesse de ne pas se retourner.

${ }^{11}$ Jakuta Alikavazovic, entretien cité (cf supra).

${ }^{12}$ Selon le titre d'un ouvrage attribué au professeur Warski. Voir le chapitre « Notes sur la conservation (4) : dans Jasper Warski, Trop voir, mal voir » (Alikavazovic, La blonde et le bunker, 49).

13 «Il la croisa plusieurs fois. Un jour, il la suivit » (16).

${ }^{14}$ Jakuta Alikavazovic, entretien cité (cf supra)

${ }^{15}$ Jakuta Alikavazovic paraphrasant Lie Tseu, article cité (cf supra).

${ }^{16}$ Selon les analyses de Jakuta Alikavazovic, entretien cité plus haut.
} 